\title{
An Exception to Contralateral Dominance of Cerebral Cortex: From Abstract to Concrete
}

\author{
Yoshikazu Isomura
}

\begin{abstract}
The cerebral cortex usually governs contralateral body parts in sensation and movements. The rule of contralateral dominance of cerebral cortex is well established on the basis of a long history of human and animal experiments. We also confirmed the rule for the primary (M1) and secondary (M2) motor cortices controlling unilateral forelimb movements in behaving rats. However, we found that their posterior parietal cortex (PPC) neurons preferentially represent ipsilateral forelimb movements, in contrast to the contralateral preference of M1 and M2 neurons. Moreover, our optogenetic activation of PPC neurons evoked ipsilaterally biased forelimb movements. Even weak PPC activation affected their task performance of ipsilateral forelimb movements. I will talk about our interpretation on these paradoxical observations from the point of view of an evolutional difference between rodents and primates.
\end{abstract}

\footnotetext{
Y. Isomura (殴)

Department of Physiology and Cell Biology, Graduate School of Medical and Dental Sciences, Tokyo Medical and Dental University, 1-5-45 Yushima, Bunkyo-ku, Tokyo 113-8510, Japan e-mail: isomura.phy2@tmd.ac.jp
} 